\title{
ONERA-NASA Cooperative Effort on Liner Impedance Eduction
}

\author{
J. Primus* ${ }^{*}$ E. Piot ${ }^{\dagger}$ and F. Simon ${ }^{\ddagger}$ \\ ONERA - The French Aerospace Lab, F-31055, Toulouse, France \\ M.G. Jones ${ }^{\S}$ and W.R. Watson $₫$ \\ NASA Langley Research Center, Hampton, VA 23681
}

\begin{abstract}
As part of a cooperation between ONERA and NASA, the liner impedance eduction methods developed by the two research centers are compared. The NASA technique relies on an objective function built on acoustic pressure measurements located on the wall opposite the test liner, and the propagation code solves the convected Helmholtz equation in uniform flow using a finite element method that implements a continuous Galerkin discretization. The ONERA method uses an objective function based either on wall acoustic pressure or on acoustic velocity acquired above the liner by Laser Doppler Anemometry, and the propagation code solves the linearized Euler equations by a discontinuous Galerkin discretization. Two acoustic liners are tested in both ONERA and NASA flow ducts and the measured data are treated with the corresponding impedance eduction method. The first liner is a wire mesh facesheet mounted onto a honeycomb core, designed to be linear with respect to incident sound pressure level and to grazing flow velocity. The second one is a conventional, nonlinear, perforate-over-honeycomb single layer liner. Configurations without and with flow are considered. For the nonlinear liner, the comparison of liner impedance educed by NASA and ONERA shows a sensitivity to the experimental conditions, namely to the nature of the source and to the sample width.
\end{abstract}

\section{Nomenclature}

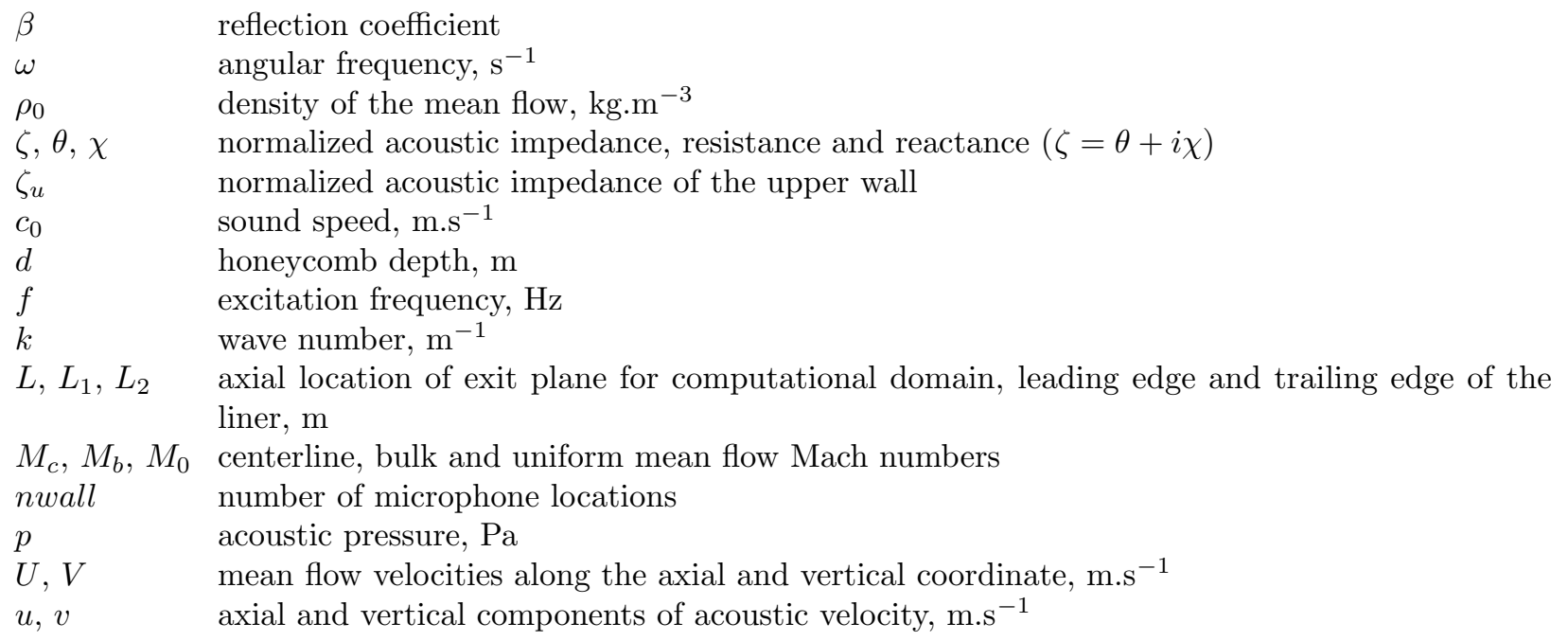

*PhD Student, ONERA DMAE-ITAC, julien.primus@onera.fr

${ }^{\dagger}$ Research Scientist, ONERA DMAE-ITAC, estelle.piot@onera.fr

${ }^{\ddagger}$ Research Scientist, ONERA DMAE-ITAC, frank.simon@onera.fr

§Senior Research Scientist, Research Directorate, Structural Acoustics Branch, AIAA Associate Fellow

ISenior Research Scientist, Research Directorate, Computational AeroSciences Branch, AIAA Associate Fellow 


$\begin{array}{ll}U_{0} & \text { uniform axial flow velocity, } \text {.s }^{-1} \\ x, y, z & \text { axial, vertical and spanwise coordinate, } \mathrm{m} \\ x_{I} & \text { axial location of microphone, } \mathrm{m} \\ \boldsymbol{A}_{x}, \boldsymbol{A}_{y} & \text { flux jacobians } \\ \boldsymbol{n} & \text { local normal vector } \\ \Gamma & \text { a domain boundary } \\ \mathcal{C} & \text { calibration coefficient } \\ \mathcal{J} & \text { objective function } \\ \Omega_{\text {obs }} & \text { observation domain } \\ \boldsymbol{\varphi} & \text { vector of acoustic perturbation, } \boldsymbol{\varphi}=(u, v, p)^{\top} \\ i & \text { unit imaginary number } \\ \cdot * & \text { adjoint quantity } \\ \left.\cdot\right|_{\text {FEM }},\left.\cdot\right|_{\text {DG }} & \text { finite element computed quantity, discontinuous Galerkin computed quantity } \\ \left.\cdot\right|_{\text {Meas }} & \text { measured quantity } \\ \cdot t, \cdot l, \cdot s & \text { quantity related to the duct termination, to the liner and to the source, respectively } \\ \bar{\cdot} & \text { complex conjugate }\end{array}$

\section{Introduction}

Since 2011, the National Aeronautics and Space Administration (NASA) and the Office National d'Etudes et de Recherches Aerospatiales (ONERA) have expressed a mutual interest in research to mitigate the environmental noise impact of civil transports during take-off and landing. One part of this cooperation addresses the issue of liner impedance eduction in the presence of grazing flow. For more than ten years, continuous effort has been provided by NASA to develop reliable impedance eduction methods (see for instance $^{1-4}$ ). A finite-length acoustic liner is mounted on a wall of a flow duct, currently the grazing flow impedance tube (GFIT), and acoustic pressure is measured by microphones flush-mounted in the wall opposite the liner. Acoustic propagation within the duct is computed by solving either the convected Helmholtz (CHE) or linearized Euler (LEE) equations, via the finite element method. The liner impedance eduction method is based on iterating the liner impedance until the acoustic pressures predicted by the finite element method match the acoustic pressure measured at a number of microphone locations to within an acceptable tolerance. Recently, ONERA has developed its own impedance eduction method, ${ }^{5-8}$ based on non-intrusive Laser Doppler Anemometry (LDA) measurements of the acoustic velocity field above the liner surface, in an aero-thermo-acoustic bench (B2A). A discontinuous Galerkin (DG) code is used to solve the linearized Euler equations, in which the mean flow profile is known from LDA measurements. Liner impedance is obtained by minimizing the difference between the computed and measured acoustic velocity field. The minimization of the objective function is achieved by the resolution, at each iteration on the liner impedance, of the direct and adjoint equations.

The purpose of the current cooperation between NASA and ONERA is to conduct tests on acoustic liners in both flow ducts (GFIT and B2A), apply the NASA and ONERA impedance eduction techniques, and gain physical and technical knowledge from comparisons of results and methodologies. Two acoustic liners have been selected, one designed to be linear with respect to source sound pressure level and grazing flow velocity (wire mesh sheet mounted onto honeycomb cells) and the other designed to be nonlinear (conventional perforate-over-honeycomb single layer liner).

In the first part of the paper, the tested liners are described, and the impedance eduction methods and flow ducts used by NASA and ONERA during this cooperation are presented. Then, results on both linear and nonlinear liners are detailed. The linear liner study is based on wall pressure measurements, while the nonlinear liner case allows comparison of the pressure and LDA approaches for impedance eduction.

\section{Impedance Eduction Methodologies}

\section{II.A. Test Liners}

Two acoustic liners have been selected, one designed to be linear with respect to source sound pressure level and grazing flow velocity, the other designed to be nonlinear. The linear liner is made of a wire mesh 
facesheet mounted onto honeycomb cells as depicted in figure 1. The facesheet is the one labeled as R2 in a

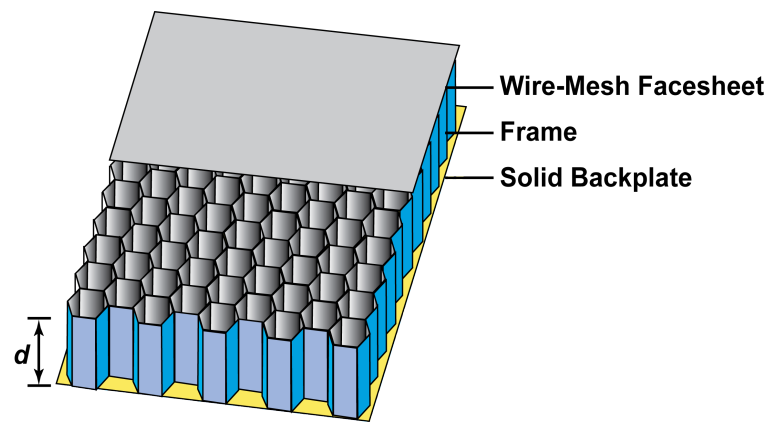

Figure 1. Sketch of wire mesh liner.

previous paper by Jones \& Watson. ${ }^{3}$ It was designed by the manufacturer to provide a DC flow resistance of 270 MKS Rayls $\left(\mathrm{kg} \cdot \mathrm{m}^{-2} \cdot \mathrm{s}^{-} 1\right.$ ), which is equivalent to a $0 \mathrm{~Hz}$ normalized acoustic resistance of $\theta=0.65$ (all impedance components in this paper are normalized by $\rho_{0} c_{0}$ ). Tests conducted with a Raylometer and in a normal incidence tube have shown that the DC flow resistance matches the manufacturer's specification, and that without flow the acoustic resistance is independent of frequency and equal to the DC flow resistance. The nonlinearity factor is 1.13 , which is considerably lower than the value, usually greater than 2 , typically measured with perforates used for conventional liner facesheets. Tests conducted in the GFIT ${ }^{3}$ proved that liners equipped with this wire mesh facesheet were virtually linear with respect to source sound pressure level (up to $130 \mathrm{~dB}$ ) and almost insensitive to grazing flow, up to a centerline Mach number of 0.3. Hence, they can reasonably be considered as linear liners for the purposes of this investigation.

Moreover, these experiments also showed that the wire mesh facesheet provides virtually no mass reactance, which means that the acoustic reactance of the liner is equal to the cavity reactance $\chi(f)=-\cot (k d)$, where $k=2 \pi f / c_{0}$ is the acoustic wave number and $d$ the honeycomb depth, respectively. The honeycomb core depth is chosen to be $38.1 \mathrm{~mm}$, causing the resonance of the liner to occur at about $1600 \mathrm{~Hz}$, which is near the center of the frequency range for testing in the GFIT and B2A flow ducts.

The nonlinear liner is a conventional perforate-over-honeycomb single layer liner, as shown in figure 2 . The perforated facesheet is $0.8 \mathrm{~mm}$ thick, its porosity is $5 \%$ and the hole diameter is $0.3 \mathrm{~mm}$. It is mounted onto a honeycomb core of $20 \mathrm{~mm}$ depth, consisting of $9.5 \mathrm{~mm}$-diameter cells (measured between two opposing flat surfaces of the hexagonal cell). Tests on this kind of liner have already been conducted, without flow, in

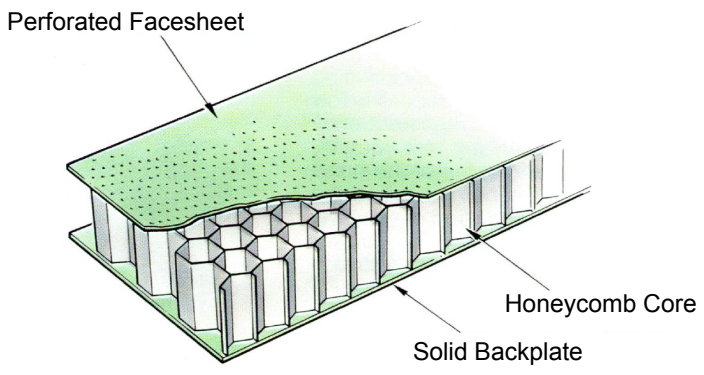

Figure 2. Sketch of conventional liner.

the ONERA B2A duct ${ }^{6,8,9}$ and for a complementary study in the normal incidence tube of LAUM. ${ }^{10}$ They showed that this micro-perforated liner is linear with respect to the source sound pressure level up to $130 \mathrm{~dB}$, but that nonlinear effects appear for greater sound pressure levels. Moreover, because of the perforations, this liner is expected to be nonlinear with respect to grazing flow velocity.

\section{II.B. Aeroacoustic Propagation Models and Impedance Eduction Methods}

\section{II.B.1. NASA Method}

An impedance eduction method based on the convected Helmholtz equation (CHE) in uniform flow is used for the current investigation. The underlying model has been presented in a previous paper, ${ }^{11}$ and is 
summarized here for convenience. Figure 3 provides a schematic of the three-dimensional flow duct and Cartesian coordinate system used in the aeroacoustic propagation model. The surface of the liner with uniform, normalized impedance, $\zeta$, comprises a portion of the upper wall of the flow duct over the axial extent of $L_{1} \leq x \leq L_{2}$. All other walls of the duct are assumed rigid, and the source and exit planes of the computational domain are located at $x=0$ and $x=L$, respectively. The sound source is assumed to be a single tone at a frequency such that no higher-order horizontal modes propagate in the rigid wall sections, thereby allowing the analysis to be restricted to the $(x, y)$-plane.

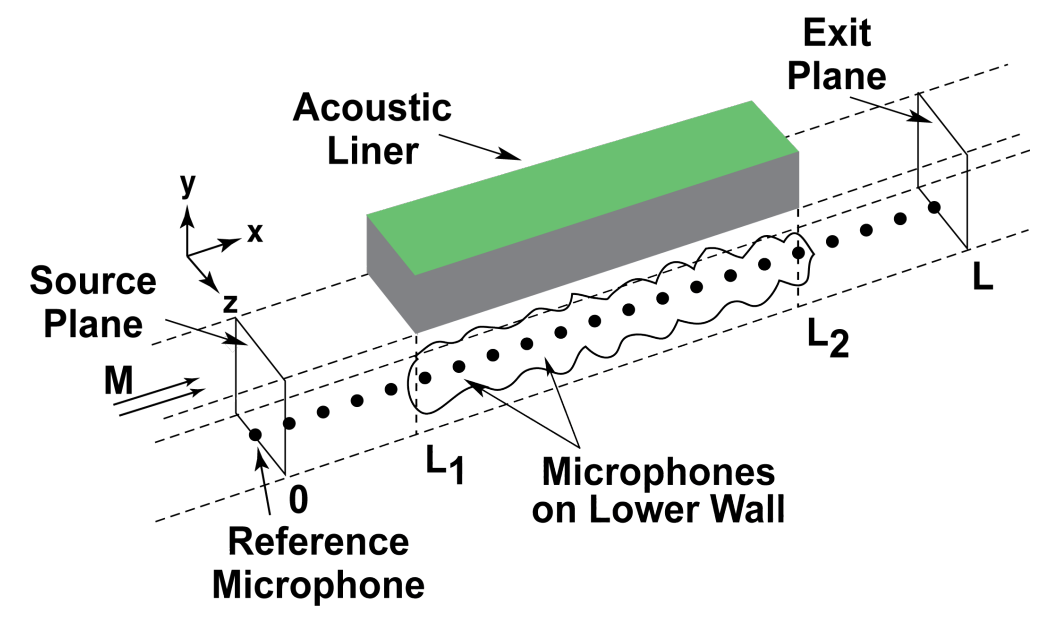

Figure 3. Sketch of Grazing Flow Impedance Tube computational domain.

Throughout the flow duct, the acoustic pressure field, $p$, is assumed to satisfy the convected Helmholtz equation

$$
\left(1-M_{0}^{2}\right) \frac{\partial^{2} p}{\partial x^{2}}+\frac{\partial^{2} p}{\partial y^{2}}-2 i k M_{0} \frac{\partial p}{\partial x}+k^{2} p=0
$$

Mean flow profiles measured just upstream and downstream of the computational domain are used to compute average axial flow velocities, and a linear fit between the two axial locations is used to determine the uniformflow axial velocity, $U_{0}$. The liner is assumed to be locally-reacting, and to be properly represented by the Myers $^{12}$ wall impedance boundary condition, given by

$$
-\frac{\partial p}{\partial y}=i k\left(\frac{p}{\zeta_{u}}\right)+2 M_{0} \frac{\partial}{\partial x}\left(\frac{p}{\zeta_{u}}\right)+\frac{M_{0}^{2}}{i k} \frac{\partial^{2}}{\partial x^{2}}\left(\frac{p}{\zeta_{u}}\right)
$$

where $\zeta_{u}$ represents the impedance of the upper wall. The admittance (i.e., $\left.1 / \zeta_{u}\right)$ is taken as zero for rigid upper wall sections of the duct located upstream and downstream of the liner, and $\zeta_{u}=\zeta$ is used in the liner portion of duct. The boundary condition of the rigid lower wall is

$$
\frac{\partial p}{\partial y}=0
$$

For a plane-wave source, the acoustic pressure profile at the source plane, $p(0, y)$, is assumed constant, and is taken to be equal to the acoustic pressure measured at the reference microphone on the lower wall, $p(0,0)$. This can be expressed as the source plane boundary condition

$$
p(0, y)=p(0,0)
$$

Similarly, the exit plane acoustic pressure boundary condition is based on the acoustic pressure measured by the microphone on the lower wall at $x=L$, and is expressed as

$$
p(L, y)=p(L, 0)
$$


Equations (1)-(5) do not have exact solutions for a general set of input parameters because the wall impedance, $\zeta_{u}$, is not constant along the wall. Consequently, they are solved numerically using a finite element method. A general purpose numerical procedure is used to determine the impedance that reproduces the acoustic pressure field measured on the wall opposite the test liner. The wall error function

$$
\mathcal{J}(\zeta)=\sum_{I=1}^{n \text { wall }}\left\{\left.p\left(x_{I}, 0\right)\right|_{\mathrm{FEM}}-\left.p\left(x_{I}, 0\right)\right|_{\text {Meas }}\right\}\left\{\left.\bar{p}\left(x_{I}, 0\right)\right|_{\mathrm{FEM}}-\left.\bar{p}\left(x_{I}, 0\right)\right|_{\text {Meas }}\right\}
$$

is used as an objective function, and the unknown liner impedance, $\zeta$, is selected such that this objective function is minimized. The overline represents the complex conjugate of the corresponding parameter. This objective function may be interpreted as the difference between the measured, $\left.p\left(x_{I}, 0\right)\right|_{\text {Meas }}$, and numerically computed, $\left.p\left(x_{I}, 0\right)\right|_{\mathrm{FEM}}$, wall pressures opposite the test liner at nwall microphone locations. The proposed procedure is perfectly general and requires only that the formulation of the duct propagation model constitutes a well-posed boundary value problem.

An initial guess of the liner impedance is required for the eduction process. For the current investigation, four independent optimizations are conducted for each test condition, each with a unique starting value of the liner impedance. These starting values are $0.5+2 i, 0.5-2 i, 2+2 i$, and $2-2 i$. A comparison of the four resultant educed impedances is used to determine whether any of the results are due to local optima. If all four values are identical, the result is generally assumed to be a global optimum. If any of the values differs from the remaining values, physical reasoning is applied to determine which one is anomalous.

\section{II.B.2. ONERA Method}

An impedance eduction method based on the two-dimensional harmonic linearized Euler equations (LEE) is used for the current investigation. The underlying model is briefly summarized here for convenience, but all details can be found in a previous paper. ${ }^{5}$ We use the same notations as in section II.B.1.

The linearized Euler equations, written under non-conservative form with a $e^{i \omega t}$ time dependence, read:

$$
\mathbf{L} \boldsymbol{\varphi}=i \omega \boldsymbol{\varphi}+\boldsymbol{A}_{x} \frac{\partial \boldsymbol{\varphi}}{\partial x}+\boldsymbol{A}_{y} \frac{\partial \boldsymbol{\varphi}}{\partial y}+\boldsymbol{B} \boldsymbol{\varphi}=0
$$

where

$$
\boldsymbol{A}_{x}=\left(\begin{array}{ccc}
U & 0 & c_{0} \\
0 & U & 0 \\
c_{0} & 0 & U
\end{array}\right), \boldsymbol{A}_{y}=\left(\begin{array}{ccc}
V & 0 & 0 \\
0 & V & c_{0} \\
0 & c_{0} & V
\end{array}\right), \boldsymbol{B}=\left(\begin{array}{ccc}
\partial_{x} U & \partial_{y} U & 0 \\
\partial_{x} V & -\partial_{x} U & 0 \\
0 & 0 & 0
\end{array}\right)
$$

Components of vector $\varphi=\left(u, v, \frac{p}{\rho_{0} c_{0}}\right)^{\top}$, where $\rho_{0}$ is the density of the mean flow, represent the acoustic perturbations around the sheared mean flow of axial and transverse velocities $U$ and $V$, respectively. The flow being assumed to be homentropic, the energy equation is replaced by the state equation $p=c_{0}^{2} \rho$, with $\rho$ the acoustic density.

As we consider a sheared mean flow profile, the impedance boundary condition on the lined wall is the standard one

$$
p= \pm \rho_{0} c_{0} \zeta v
$$

the sign depending on whether the upper or lower wall is considered. To avoid any singularities in the rigid wall case, the boundary condition (8) is expressed as a function of the normal incidence reflection coefficient $\beta=(\zeta-1) /(\zeta+1)$, leading to:

$$
\boldsymbol{M}_{\beta}=\frac{1}{2}\left(\begin{array}{cc}
(\beta+1) \boldsymbol{n} \otimes \boldsymbol{n} & (\beta-1) \boldsymbol{n} \\
-(\beta+1) \boldsymbol{n}^{\top} & (1-\beta)
\end{array}\right)=0
$$

where $\boldsymbol{n}$ is the local normal vector and $\otimes$ the tensor product. On the rigid walls the reflection coefficient $\beta$ is then taken as 1 .

At the source plane, the state vector $\varphi$ is taken to be equal to $\mathcal{C} \varphi_{0}$, where $\varphi_{0}$ is the plane-wave solution in a rigid duct with uniform flow Mach number $M_{0}$, corresponding to the bulk Mach number of the actual shear flow $U(y)$. As this plane-wave solution is chosen with an arbitrary amplitude taken to be equal to 
1 , the complex coefficient $\mathcal{C}$ stands for the source amplitude. $\mathcal{C}$ can be either set to the measured value of the acoustic pressure at the source plane, when available, or included into the eduction process to reproduce at best the measured acoustic field. The second option is chosen in this paper. At the exit plane, either a non-reflecting or an exit impedance boundary condition can be enforced. The exit impedance is denoted as $\zeta_{t}$.

A discontinuous Galerkin (DG) scheme is chosen to solve Eq. (7) and the associated boundary conditions. In this method, discontinuities are allowed at the interface between two elements. A triangular mesh of Lagrange $\mathbb{P}_{1}$ elements is used. To ensure the connection between elements and to apply the boundary conditions, a numerical flux has to be defined. An upwind numerical flux is used to ensure connection between interior cells, while a centered flux is chosen at the boundaries (see previous papers ${ }^{5,6,13}$ for more details).

The inverse problem is defined by the minimization of the following objective function:

$$
\mathcal{J}\left(\boldsymbol{\varphi}, \zeta, \zeta_{t}, \mathcal{C}\right)=\int_{\Omega_{o b s}}\left\{\left.\boldsymbol{\varphi}\right|_{\mathrm{DG}}-\left.\boldsymbol{\varphi}\right|_{\text {Meas }}\right\}^{\top}\left\{\left.\bar{\varphi}\right|_{\mathrm{DG}}-\left.\overline{\boldsymbol{\varphi}}\right|_{\text {Meas }}\right\} \mathrm{d} x \mathrm{~d} y
$$

where $\Omega_{o b s}$ is the observation region. In this paper, two different configurations are considered, illustrated in figure 4 . When the impedance eduction method is based on acoustic pressure measurements on the wall opposite the test liner (see figure $4(\mathrm{a})$ ), $\Omega_{o b s}$ is made of the discrete set of microphone locations at the wall, and only the pressure component of the state vector is considered in the error function (10). On the contrary, when the impedance eduction method is based on LDA measurements above the test liner (see figure 4(b)), $\Omega_{o b s}$ is a rectangular $(x, y)$ region and the $(u, v)$ velocity components of the state vector are considered in $(10)$.

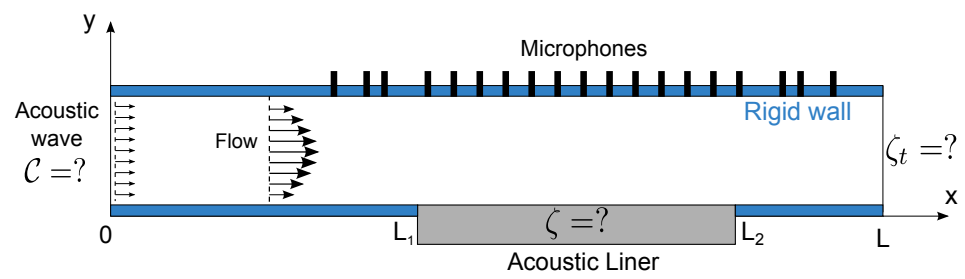

(a) Impedance eduction from microphone measurements

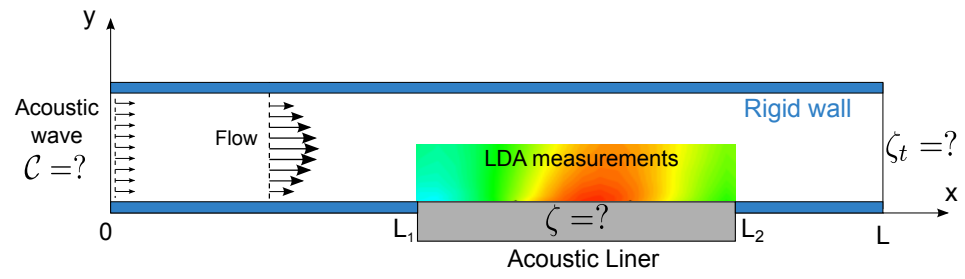

(b) Impedance eduction from LDA measurements

Figure 4. Sketch of both configurations on which ONERA impedance eduction method is applied.

A BFGS-B (Broyden, Fletcher, Goldfarb, Shanno for bounded variables) algorithm is used to solve the optimization problem. ${ }^{14}$ The analytical expression of the gradient of $\mathcal{J}$ with respect to each of the search parameters $\zeta, \zeta_{t}, \mathcal{C}$ is obtained via an adjoint method, as explained in Primus et al.:. ${ }^{5}$

$$
\begin{aligned}
\frac{\partial \mathcal{J}}{\partial \zeta} & =-\left\langle\frac{\partial \boldsymbol{M}_{\beta}}{\partial \beta} \frac{\partial \beta}{\partial \zeta} \boldsymbol{\varphi}, \boldsymbol{\varphi}^{*}\right\rangle_{\Gamma_{l}} \\
\frac{\partial \mathcal{J}}{\partial \zeta_{t}} & =-\left\langle\frac{\partial \boldsymbol{M}_{\beta_{t}}}{\partial \beta_{t}} \frac{\partial \beta_{t}}{\partial \zeta_{t}} \boldsymbol{\varphi}, \boldsymbol{\varphi}^{*}\right\rangle_{\Gamma_{t}} \\
\frac{\partial \mathcal{J}}{\partial \mathcal{C}} & =\left\langle\boldsymbol{A}^{i} n_{i}^{-} \boldsymbol{\varphi}_{0}, \boldsymbol{\varphi}^{*}\right\rangle_{\Gamma_{s}}
\end{aligned}
$$

where $\boldsymbol{\varphi}^{*}$ is the solution of the adjoint problem and $\boldsymbol{A}^{i} n_{i}^{-}$is the matrix associated to the negative eigenvalues of the matrix $\boldsymbol{A}^{i} n_{i}=\boldsymbol{A}_{x} \boldsymbol{n}_{x}+\boldsymbol{A}_{y} \boldsymbol{n}_{y} . \Gamma_{l}, \Gamma_{t}$ and $\Gamma_{s}$ are the liner surface, the exit plane and the source plane, respectively. A formulation entirely based on $\beta$ could have been chosen, but with this $\zeta$-approach for the inverse problem, the positivity of the resistance can easily be ensured. 


\section{II.C. Description of the Flow Ducts}

\section{II.C.1. NASA Grazing Flow Impedance Tube}

The NASA impedance eduction method is currently implemented using data acquired in the grazing flow impedance tube (GFIT). This waveguide has a cross-sectional geometry of $50.8 \mathrm{~mm} \times 63.5 \mathrm{~mm}$, such that higher-order modes in the horizontal and vertical dimensions cut on at different frequencies, and allows evaluation of acoustic liners with lengths from $50.8 \mathrm{~mm}$ to $609.6 \mathrm{~mm}$. As shown in Fig. 5, flow propagates from left to right. This flow is achieved via the combination of an upstream high pressure air supply and a downstream vacuum blower, such that the test window containing the acoustic liner can be exposed to near-ambient pressure conditions for mean flows up to Mach 0.6. The surface of the test liner forms a portion of the upper wall of the flow duct. Eighteen acoustic drivers are mounted upstream of the test window, and are used to generate tones (one frequency at a time) at up to $150 \mathrm{~dB}$ over a frequency range of 0.4 to $3.0 \mathrm{kHz}$.

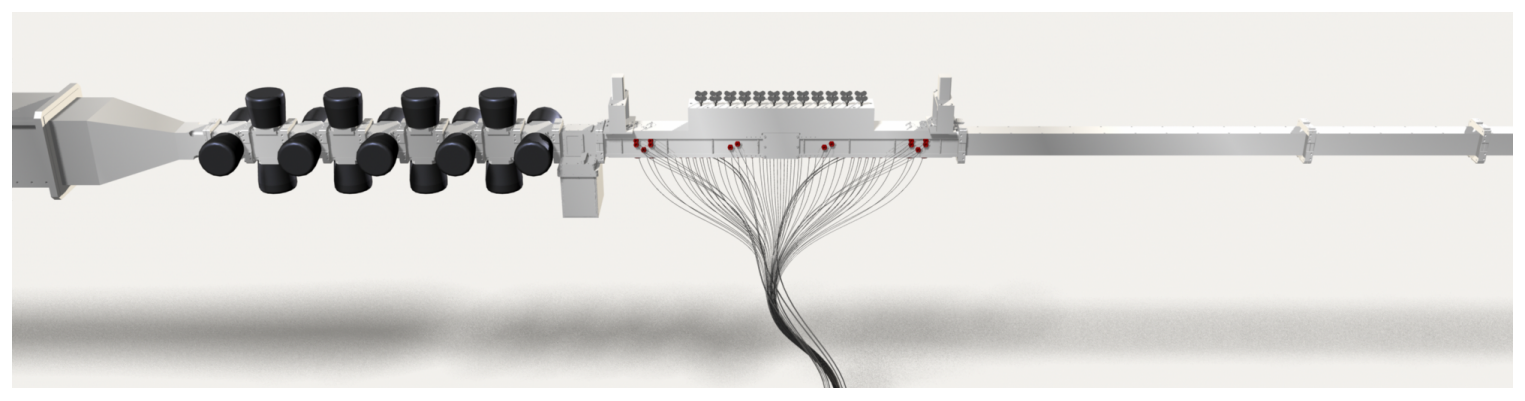

Figure 5. Sketch of Grazing Flow Impedance Tube.

Fifty-three microphones are flush-mounted in the lower wall (opposite the liner) to measure the acoustic pressure field from upstream of the liner leading edge to downstream of the liner trailing edge. Each liner is tested using a tonal source (one frequency at a time), with source sound pressure levels up to $140 \mathrm{~dB}$, and at frequencies of 0.4 to $3.0 \mathrm{kHz}$ in $0.2 \mathrm{kHz}$ increments. For each data acquisition, 1000 averages (blocks of 1024 data points per average) are acquired with each microphone. To reduce the influence of flow noise, a cross-spectrum signal extraction method ${ }^{15}$ is used to determine the amplitudes and phases at each of the microphone locations relative to the amplitude and phase at the reference microphone location.

A dual-axis traverse probe system is used to acquire flow profiles upstream and downstream of the liner. Two pitot probes are used for this measurement, to allow detailed measurements close to the upper and lower walls of the GFIT. The traverse system uses two stepping motors to successively move the pitot probe to preselected measurement locations, such that the full flow profile can be accurately determined. The traverse probe system is removed for acoustic measurements.

\section{II.C.2. ONERA B2A Duct}

The aeroacoustic test bench at Onera is made of a stainless steel tube of section $50 \mathrm{~mm}$ x $50 \mathrm{~mm}$; its total length is about $4 \mathrm{~m}$. A $0.2 \mathrm{~m}$-long test section is equipped with two silica windows for optical access. The termination is equipped with a quasi-anechoic outlet, leading to upstream reflection coefficient smaller than 0.2 for frequencies higher than $500 \mathrm{~Hz}$. A mean flow of bulk Mach number up to 0.3 can be provided. In the test section, this flow shows fully developed turbulent boundary layers, with a turbulence rate of a few percent at the center of the test section. As shown in Fig. 6(a), flow propagates from left to right. Two acoustic drivers are mounted upstream of the test section, and are used to generate tones (usually multi-sine signal) at up to $140 \mathrm{~dB}$ over a frequency range of 0.3 to $3.5 \mathrm{kHz}$ (i.e., the no-flow cut-off frequency of the duct). As shown in Fig 6(b), the surface of the test liner forms a $150 \mathrm{~mm}$-long portion of the lower wall of the flow duct. It should be noted that the liner spans only three-fifth of the duct width (i.e., $10.16 \mathrm{~mm}$-wide hard wall strips are present on either side).

Sixteen microphone locations are available in the upper wall (opposite the liner) to measure the acoustic pressure field from upstream of the liner leading edge to downstream of the liner trailing edge. Flush-mounted B\&K 4182 microphone probes are used (probe diameter of $1.2 \mathrm{~mm}$ ). Usually, only two upstream locations are 


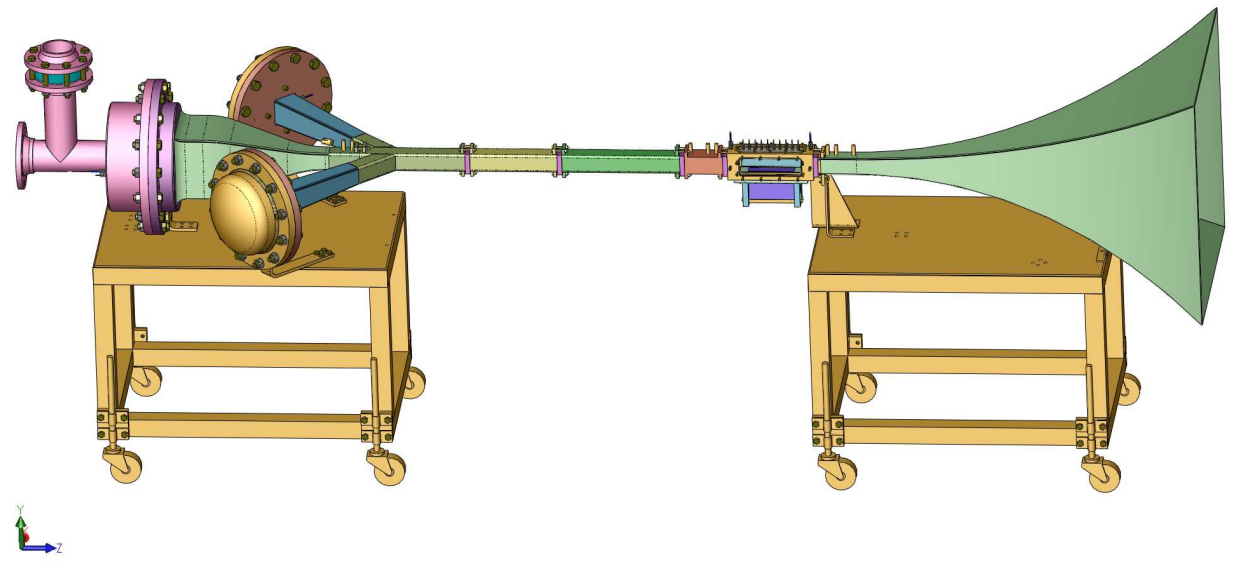

(a) Global view of B2A duct

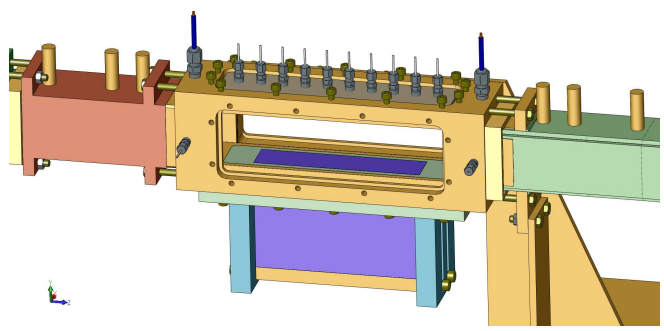

(b) Zoom on the testing cell. The acoustic liner is in purple.

Figure 6. Sketch of B2A Aero-Thermo-Acoustic test bench. 
considered to measure the amplitude of the incident acoustic plane waves. ${ }^{16}$ However, for the pressure-based impedance eduction method (as displayed in Fig. 4(a)), one microphone probe is moved along the whole set of microphone locations. The cross-spectrum between the microphone and driver signal is acquired, which provides a phase reference for the pressure measurements.

A two-component fringe mode Laser Doppler Velocimeter allows the measurement of the axial and transverse velocity components in almost the entire volume of the test section. For the velocity-based impedance eduction methods (as displayed in Fig. 4(b)), only the central plane $\mathrm{z}=25 \mathrm{~mm}$ is scanned. The emitting optics produce a $100 \mu$ m-diameter measurement volume. Flow is seeded with either incense smoke or amorphous silica particles. Consequently, the LDA signal is unevenly sampled due to the random arrival of particles in the measurement volume. A reconstruction method ${ }^{17}$ is used to re-sample the raw data at a constant rate. Data processing is performed by a TSI IFA 755 system. A minimum sampling data rate of $f_{m}=13000$ measurements per second is generally ensured, for each velocity component.

Each velocity component $u$ and $v$ is measured by the LDA system at a given spatial location $(x, y)$. The acoustic velocity is defined here as the component of the signal that is correlated with the driver signal. It can be educed from the extraneous noise (mainly due to the turbulent flow) by a technique similar to the three-microphone signal enhancement technique. ${ }^{16,18}$ It consists in calculating the cross-spectral density function $G_{V_{i}, l s}$ between the velocity signal $V_{i}$ and the loudspeaker signal $l s\left(\mathrm{see}^{17,19,20}\right)$. The auto-spectral density function of the acoustic velocity reads:

$$
G_{v_{i}}=\frac{\left|G_{V_{i}, l s}\right|^{2}}{G_{l s}}
$$

where $G_{l s}$ is the auto-spectral density function of the loudspeaker signal. The acoustic velocity in the frequency domain is then given by

$$
v_{i}=\sqrt{G_{v_{i}}} \exp \left[i \Phi\left(V_{i} / l s\right)\right]
$$

where the phase of the acoustic velocity, referenced by the loudspeaker, is defined as

$$
\Phi\left(V_{i} / l s\right)=\arctan \frac{\operatorname{Im}\left(G_{V_{i}, l s}\right)}{\operatorname{Re}\left(G_{V_{i}, l s}\right)} .
$$

\section{Comparison of Impedance Eduction Results}

\section{III.A. Linear Wire Mesh Liner}

The wire mesh liner has already been tested by NASA in the GFIT duct. Results of the impedance eduction method have been published by Jones \& Watson, ${ }^{3}$ without and with flow. For the present study, a sample of the wire mesh liner was built for the B2A duct. Tests have been conducted by ONERA, without and with flow. Because of the small size of the wire mesh openings, the seeding particles used for LDA measurements can quickly reduce the sample equivalent porosity and thus modify the liner impedance. As a consequence, for this particular treatment, LDA has only been used to measure the mean flow profile $(U, V)$ in the duct.

As these contaminating issues were a matter of concern, we first performed microphone measurements at the wall opposite the clean liner, as explained in sections II.B.2 and II.C.2. Results of the pressure-based impedance eduction method are compared to NASA published results ${ }^{3}$ in figure 7 . The centerline Mach number is $M_{c}=0.2$ in ONERA experiments while NASA experiments have been conducted with $M_{c}=0.3$. However, as the liner is virtually linear with respect to flow velocity, we can expect that this difference in flow velocity does not have a significant influence on the eduction results. The value of the DC flow resistance is plotted for comparison, as well as the cavity reactance $\chi(f)=-\cot (k d)$.

Without flow, results obtained in the ONERA duct show the correct behavior, but with a slight underestimation of the reactance compared to NASA and theoretical values. For frequencies below $1 \mathrm{kHz}$ and above $2 \mathrm{kHz}$, the acoustic liner provides quite small attenuation, especially in the B2A duct since the length of the liner is much smaller than in the GFIT. This is illustrated by figure 8 , which shows the SPL measurements at 2800 or $2880 \mathrm{~Hz}$ where the attenuation is low. The acquired pressure at $1600 \mathrm{~Hz}$, where the decrease is sufficient for impedance evaluation, is also shown for comparison. As a result this leads to an uncertainty much larger than in the $1 \mathrm{kHz}-2 \mathrm{kHz}$ frequency range, resulting in unreliable educed impedance from the B2A set of measurements.

With flow, the educed impedance is similar to the one without flow, as expected for such a linear liner, but the ONERA results are less smooth. The previous remark holds even more in the presence of flow with 

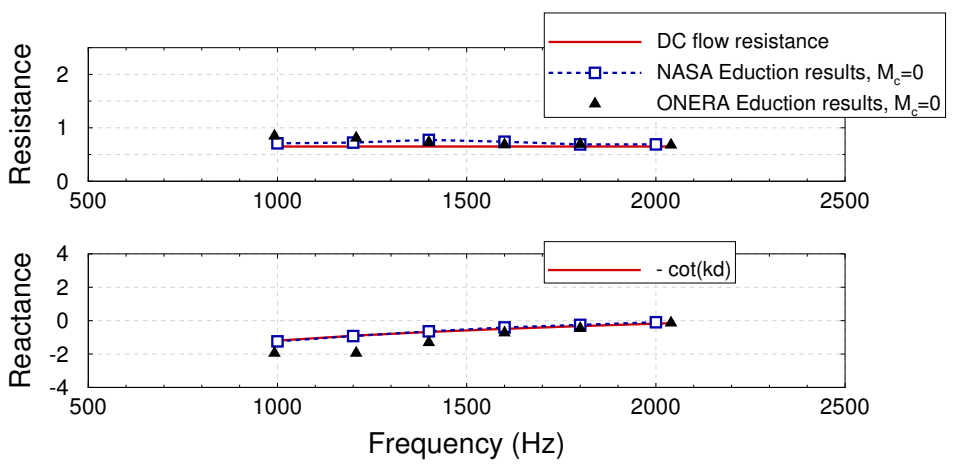

(a) Without flow
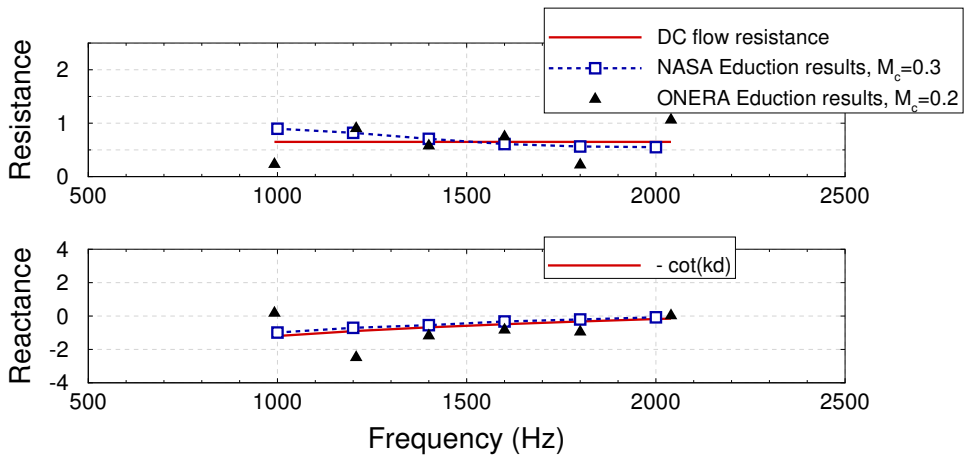

(b) With flow

Figure 7. Educed impedance from ONERA experiments compared to NASA results, for the linear liner.

an upstream acoustic source, as in that case the wavelength is longer than without flow. Hence the ratio of liner length per wavelength diminishes in consequence, and the attenuation properties of the linear liner are reduced. The scattering of the ONERA impedance can also be explained by the small amount of measured data in the B2A duct (recall that the liner length in the B2A is much less than in the GFIT), which yields to an increase of the uncertainty in the impedance eduction technique.

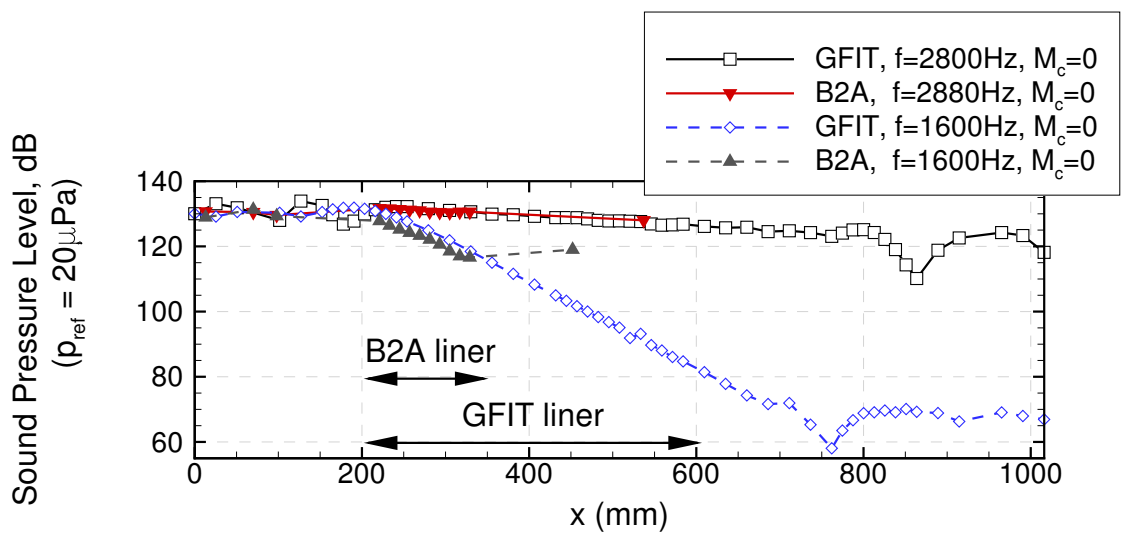

Figure 8. SPL values measured at the wall opposite the liner, at $2880 \mathrm{~Hz}$ or $2800 \mathrm{~Hz}$ and at $1600 \mathrm{~Hz}$ without flow, in both B2A and GFIT ducts.

The ONERA impedance eduction technique has also been directly applied to the GFIT set of pressure measurements without flow and with a flow of centerline Mach number $M_{c}=0.3$ (bulk Mach number $\left.M_{b}=0.253\right)$. In the latter case the flow profile has been acquired upstream and downstream of the liner with a pitot probe as described in section II.C.1. A mean sheared flow profile is deduced from these measurements and used in the ONERA impedance eduction code (figure 9), while a uniform flow is assumed in the NASA 
eduction code.

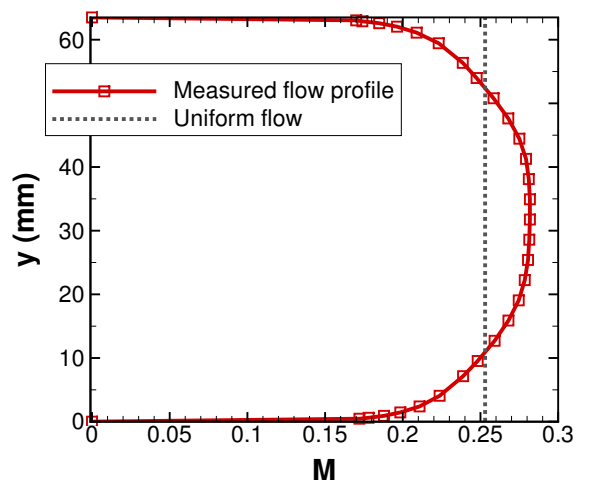

Figure 9. Mean flow profile over the linear wiremesh liner, measured by pitot probe in the GFIT.

Impedances educed with the ONERA technique are plotted in figure 10, without and with flow, and compared to impedances educed by NASA on the same set of measured acoustic pressure. The upper bound of the frequency range is determined by the cut-on frequency of the first higher-order mode with flow. Educed
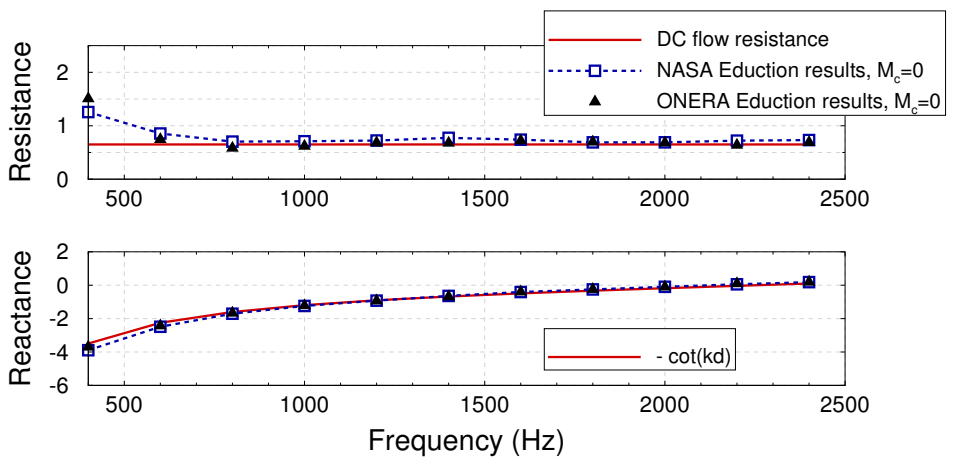

(a) Without flow
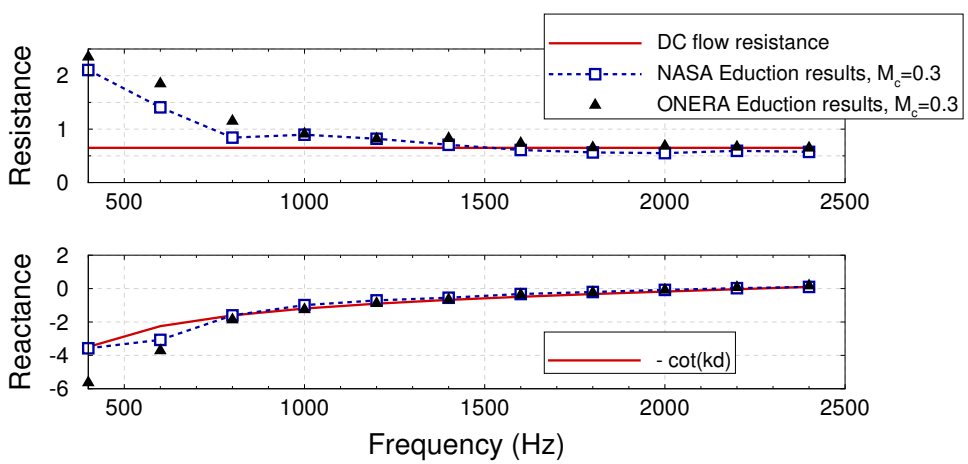

(b) With flow $\left(M_{c}=0.3\right)$

Figure 10. Educed impedance from ONERA method applied to NASA data, compared to NASA results, for the linear liner.

impedances from the ONERA method match almost perfectly with NASA results both on resistance and reactance. This confirms that the disparity between ONERA and NASA educed values in figure 7 are due to differences in geometry and experimental conditions between the B2A bench and the GFIT, and not to the impedance eduction code by itself. In particular, NASA and ONERA impedances are very similar when educed on GFIT data with flow (figure 10). The only noteworthy differences in this case arise in low frequencies, and are likely due to the consideration of a sheared flow profile as opposed to the uniform flow assumption. These results confirm that the quite strong uncertainty observed with flow in figure 7 is due to the small size of the sample and of the measured dataset in the B2A duct. 


\section{III.B. Nonlinear Conventional Liner}

Experiments have been conducted in the B2A, without flow and with a bulk flow Mach number $M_{b}=0.2$, i.e. a centerline Mach number $M_{c}=0.23$. Eight harmonic acoustic plane waves are emitted, from $f=992 \mathrm{~Hz}$ to $f=3136 \mathrm{~Hz}$. The incoming sound pressure level relative to each pure tone is either $120 \mathrm{~dB}$ or $130 \mathrm{~dB}$, which corresponds to an overall sound pressure level (OASPL) of $130 \mathrm{~dB}$ and $140 \mathrm{~dB}$, respectively. The measurement area is located above the liner up to an ordinate $y=30 \mathrm{~mm}$. In the $x$-axis direction, a left and a right measurement margin of $7 \mathrm{~mm}$ each surround the leading and trailing edge of the liner, giving a measurement region spreading over $x \in\left[L_{1}-7 ; L_{1}+157\right] \mathrm{mm}$.

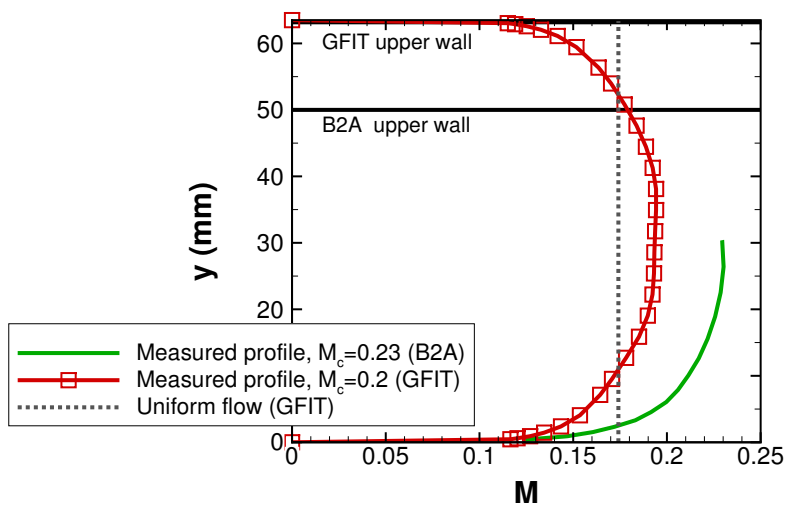

Figure 11. Mean flow profile over the nonlinear liner measured by LDA in the B2A duct and measured by pitot probe in the GFIT.

The nonlinear liner has also been tested in the GFIT, without flow and with a bulk flow Mach number $M_{b}=0.174$, i.e. a centerline Mach number $M_{c}=0.2$ (see figure 11). Note that the slight difference in the flow velocities between the GFIT and B2A experiments is likely to impact the impedance of this nonlinear liner. It should also be remembered that a tonal source is used in the GFIT, as described in section II.C.1: the harmonic waves are emitted one by one, with a sound pressure level of either $120 \mathrm{~dB}$ or $130 \mathrm{~dB}$. The nature of the source thus differs from the B2A, where a multi-sine source is used. Furthermore, the choice is made in the B2A to impose the level of the incoming wave only, the wave reflected at the leading edge of the liner being sorted and put aside. In the GFIT, it has been chosen to impose a total level taking into account the incident and reflected waves. Finally it must be noted that, contrary to the GFIT sample, the B2A sample does not span the whole width of the duct, but only three-fifth of it. To mimic this configuration, some additional tests have been run in the GFIT with rigid margins placed along the nonlinear liner sample. The $50.8 \mathrm{~mm}$ width of the GFIT material has thus been partially diminished by the addition of $10.16 \mathrm{~mm}$-wide strips on either side.

The impedance values educed with NASA and ONERA methods are compared in figure 12 for the case without flow. The ONERA results show an important sensitivity of the resistance to the incident sound pressure level, whereas it has almost no influence on the NASA educed values. Moreover the resistance found with the ONERA approach is much higher than with the NASA technique, and the reactance much lower. Although not represented on the graph for the sake of clarity, impedance tube measurements with a white noise source lead to a reactance curve situated between the NASA and ONERA ones on the studied frequency range. ${ }^{10}$ Two combined hypotheses can be made to explain these behaviors. The first one relates to the different type of source employed in the benches. Indeed, with the use of a multi-sine source at sufficiently high sound pressure levels, nonlinear interactions between the excited frequencies can occur and affect the absorption properties of the liner. ${ }^{21}$ This implies an increase in the resistance and a decrease in the reactance compared to a single tone excitation. The second hypothesis, related to the increase of resistance only, concerns the width of the sample. The presence of rigid parts along the liner can result in a reduction of the absorption, which expresses itself by a higher educed resistance. This is confirmed by the resistance educed in the GFIT with a sample of reduced width. The reactance is not significantly affected, since the main reactive contribution comes from the honeycomb cavities. The multiplying factor corresponding to the rise in resistance can be evaluated with a simple reasoning, by considering that the sample with rigid strips is equivalent to a sample occupying the whole width of the duct but with a reduced perforated surface. Its equivalent porosity is then three-fifth of the original sample without strips. It can be checked that the 
resistance is approximately multiplied by $5 / 3$ with the addition of rigid strips. Finally, higher-order modes may appear in the spanwise direction owing to the impedance discontinuities between the rigid and lined parts.

Nevertheless the effect of rigid margins is not visible on the impedance educed with the ONERA process applied to acoustic pressure measurements in the B2A (see section III.A). Further evaluation has to be carried out for a better understanding of this result.
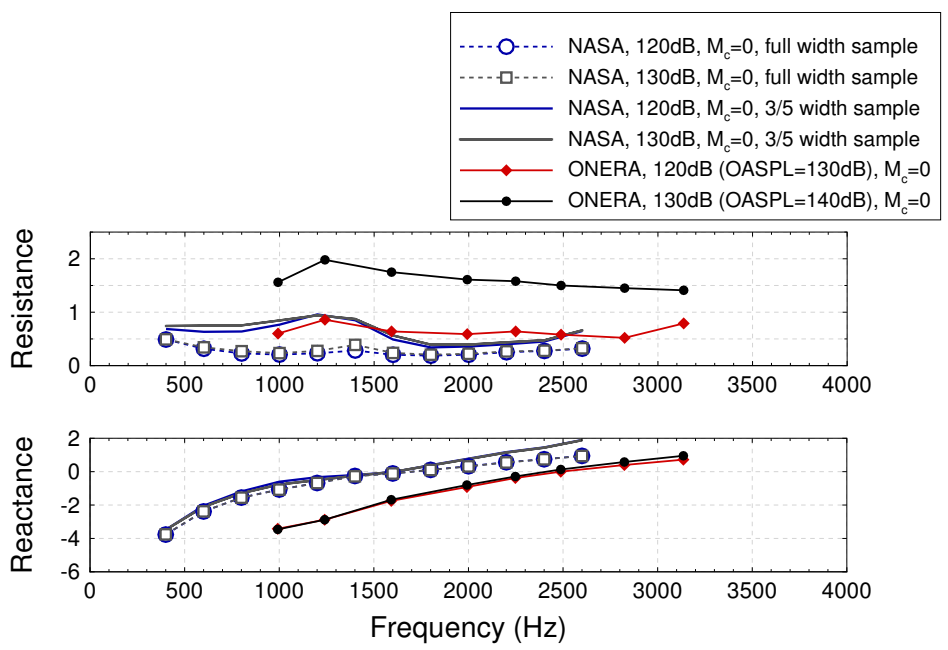

Figure 12. Educed impedance from NASA and ONERA experiments on the nonlinear liner, in the absence of flow.

In the presence of flow, the sensitivity of resistance to incident level for the ONERA method is decreased compared to the case without flow (figure 13). The flow tends to linearize the behavior of the liner with respect to sound pressure level. The fact that the impedances educed by NASA tend to get closer to the ONERA values would then comfort the idea that the nature of the acoustic excitation has a significant influence on the liner impedance. Also, the presence of flow does not change significantly the reactance.

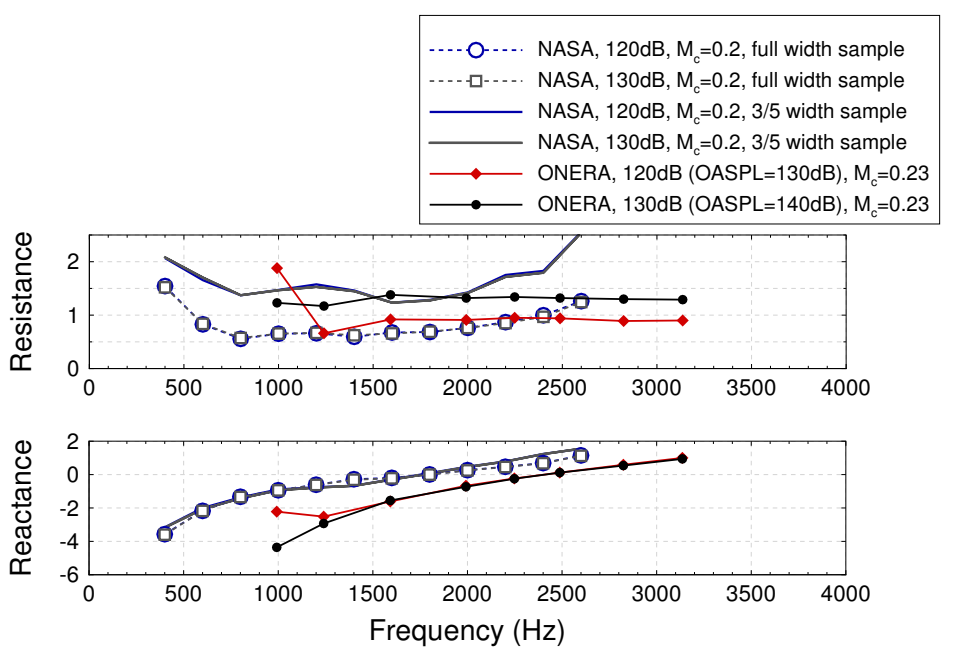

Figure 13. Educed impedance from NASA and ONERA experiments on the nonlinear liner, in the presence of flow.

\section{Concluding Remarks}

A comparison has been made between the methodologies developed at NASA Langley and ONERA for the eduction of liner impedance. Both procedures have been applied to a wire mesh liner, which is considered linear with respect to incident acoustic level and mean flow, and to a conventional perforate-over-honeycomb 
single layer liner with a nonlinear behavior.

A first observation is that the application of the ONERA eduction code on acoustic pressure measurements made in the NASA test facility leads to an impedance spectrum very close to the one obtained with the NASA eduction code, which confirms the results of previous papers. ${ }^{5-8}$ The only differences arising appear in the low frequency range in the presence of flow, and are attributed to the consideration of the shear layer of the mean flow. This suggests that the numerical methods used at NASA and ONERA can be considered equivalent, at least concerning the obtained impedances; other properties such as convergence speed remain to be studied.

In the case of the linear liner, the impedance identified from pressure measurements in the NASA GFIT and ONERA B2A show small but non-negligible differences, especially with flow. These were imputed to the lack of measured data in B2A which, associated to the relatively short length of the liner, prevent the reliability of the numerical identification method to be as good as with the NASA measurements. This result was foreseeable as the $\mathrm{B} 2 \mathrm{~A}$ test bench was not initially designed to perform such acoustic pressure measurements. However, in the frequency range where the absorption of the liner is sufficient, the results are quite promising. Finally, the differences in the educed impedance of the nonlinear liner are likely due to the nature of the source (single-sine versus multi-sine) and to the sample width, the nonlinear interactions between the excited frequencies of the multi-sine source and the presence of rigid strips along the sample tending to increase the educed resistance. The use of the multi-sine source also seems to decrease the reactance. Further study has to be carried out to gain insight on these matters. For instance, the sensitivity to the sample width could be numerically evaluated by taking into account the third dimension in the propagation code and considering a partially lined duct width, with impedance discontinuities in the $z$ direction likely to scatter acoustic energy into higher-order spanwise modes. A modification of the B2A test cell to receive a full width sample is also planned. Regarding the source, experiments with both mono-sine and multi-sine sources in a same test bench are to be considered. Last but not least, complementary acoustic pressure measurements will be performed in B2A at the rigid wall opposite the nonlinear liner in order to understand why the aforementioned effects could not be observed with pressure measurements opposite the linear wire mesh liner.

\section{References}

\footnotetext{
${ }^{1}$ Brown, M. C., Jones, M. G., and Watson, W. R., "Uncertainty Analysis of the Grazing Flow Impedance Tube," Proceedings of the 18th AIAA/CEAS Aeroacoustics Conference, AIAA 2012-2296, Colorado Springs, Colorado, USA, 4-6 June 2012.

${ }^{2}$ Watson, W. R. and Jones, M. G., "Evaluation of Wall Boundary Conditions For Impedance Eduction Using a Dual-Source Method," Proceedings of the 18th AIAA/CEAS Aeroacoustics Conference, AIAA 2012-2199, Colorado Springs, Colorado, USA, 4-6 June 2012.

${ }^{3}$ Jones, M. G. and Watson, W. R., "On the Use of Experimental Methods to Improve Confidence in Educed Impedance," Proceedings of the 17th AIAA/CEAS Aeroacoustics Conference, AIAA-2011-2865, Portland, Oregon, 5-8 June 2011.

${ }^{4}$ Watson, W. R., Jones, M. G., and Gerhold, C. H., "Implementation and Validation of an Impedance Eduction Technique," Proceedings of the 17th AIAA/CEAS Aeroacoustics Conference, AIAA-2011-2867, Portland, Oregon, 5-8 June 2011.

${ }^{5}$ Primus, J., Piot, E., and Simon, F., "An adjoint-based method for liner impedance eduction: Validation and numerical investigation," J. Sound Vib., Vol. 332, No. 1, January 2013, pp. 58-75.

${ }^{6}$ Piot, E., Primus, J., and Simon, F., "Liner impedance eduction technique based on velocity fields," Proceedings of the 18th AIAA/CEAS Aeroacoustics Conference, AIAA 2012-2198, Colorado Springs, Colorado, USA, 4-6 June 2012.

${ }^{7}$ Primus, J., Piot, E., and Simon, F., "Evaluation of an adjoint-based liner impedance eduction technique," Proceedings of the Acoustics 2012 Nantes Conference, Nantes, France, 23-27 April 2012.

${ }^{8}$ Primus, J., Simon, F., and Piot, E., "Validation of a direct propagation model for liner impedance eduction," Proceedings of the 17th AIAA/CEAS Aeroacoustics Conference, AIAA-2011-2869, Portland, Oregon, June 2011.

${ }^{9}$ Nennig, B., Tahar, M. B., Ville, J. M., Piot, E., Simon, F., and Taktak, M., "A comparison between acoustic liner characterization methods and calculations," Proceedings of the 16th Congress on Sound and Vibration, ICSV, July 2009.

${ }^{10}$ Escouflaire, M., Redonnet, S., and Aurégan, Y., "Numerical Prediction Of Absorbing Materials Via Computational AeroAcoustics," Proceedings of InterNoise 2012, New York City, USA, 19-22 August 2012.

${ }^{11}$ Watson, W. R. and Jones, M. G., "Impedance Eduction in Ducts with Higher-Order Modes and Flow," AIAA Paper 2009-3236, May 2009.

${ }^{12}$ Myers, M. K., "On the Acoustic Boundary Condition in the Presence of Flow," Journal of Sound and Vibration, Vol. 71, No. 3, 1980, pp. 429-434.

${ }^{13}$ Pascal, L., Piot, E., and Casalis, G., "Discontinuous Galerkin Method for acoustic modes computation in lined ducts," Proceedings of the 18th AIAA/CEAS Aeroacoustics conference, AIAA 2012-2153, Colorado Springs, Colorado, USA, 4-6 June 2012.

${ }^{14}$ Byrd, R. H., Nocedal, J., and Schnabel, R. B., "A limited memory algorithm for bound constrained optimization," SIAM Journal on Scientific and Statistical Computing, Vol. 16, 1995, pp. 1190-1208.
} 
${ }^{15}$ Bendat, J. S. and Piersol, A. G., Random Data: Analysis and Measurement Procedures, Wiley-Interscience, 1971.

${ }^{16}$ Chung, J. Y. and Blaser, D. A., "Transfer function method of measuring in-duct acoustic properties. I. Theory," J. Acoust. Soc. Am., Vol. 68 (3), 1980, pp. 907-913.

${ }^{17}$ Minotti, A., Simon, F., and Gantié, F., "Characterization of an acoustic liner by means of Laser Doppler Velocimetry in a subsonic flow," Aerospace Science and Technology, Vol. 12, No. 5, 2008, pp. 398-407.

${ }^{18}$ Nance, D. and Ahuja, K. K., "Limitations of the three-microphone signal enhancement technique," Proceedings of the 45th AIAA Aerospace Sciences Conference, AIAA-2007-441, Reno, Nevada, January 2007.

${ }^{19}$ Lavieille, M., Simon, F., and Micheli, F., "Measurement of acoustic quantity fields in duct flow by Laser Doppler Velocimetry." Proceedings of the 12th AIAA/CEAS Aeroacoustics Conference, AIAA-2006-2550, Cambridge, Massachusetts, May 8-10 2006.

${ }^{20}$ Betgen, B., Galland, M. A., Piot, E., and Simon, F., "Implementation and non-intrusive characterization of a hybrid active-passive liner with grazing flow," Applied Acoustics, Vol. 73, 2012, pp. 624-638.

${ }^{21}$ Bodén, H., "The effect of high level multi-tone excitation on the acoustic properties of perforates and liner samples," Proceedings of the 18th AIAA/CEAS Aeroacoustics Conference, AIAA 2012-2151, Colorado Springs, Colorado, USA, 4-6 June 2012. 